\title{
The Prevalence of Physical Inactivity in Iranian Children and the Impact of Economic and Social Inequalities on it: Results of a National Study in 2018
}

\section{Ghobad Moradi}

Kurdistan University of Medical Sciences

Farideh Mostafavi

Kurdistan University of Medical Sciences

Bakhtiar Piroozi ( $\nabla$ bpiroozi@gmail.com )

Kurdistan University of Medical Sciences

Bushra Zareie

Kurdistan University of Medical Sciences

Marzieh Mahboobi

Tehran University of Medical Sciences

Mohammad Aziz Aziz Rasouli

Kurdistan University of Medical Sciences

\section{Research}

Keywords: Socioeconomic status, Inequalities, Physical inactivity, Concentration index, Iran

Posted Date: April 15th, 2020

DOI: https://doi.org/10.21203/rs.3.rs-22673/v1

License: (c) (i) This work is licensed under a Creative Commons Attribution 4.0 International License.

Read Full License 
The Prevalence of Physical Inactivity in Iranian Children and the Impact of Economic and Social Inequalities on it: Results of a National Study in 2018

Ghobad Moradi ${ }^{1}$, Farideh Mostafavi ${ }^{1}$, Bakhtiar Piroozi ${ }^{1}$, Bushra Zareie ${ }^{1,2}$, Marzieh Mahboobi ${ }^{3}$, Mohammad Aziz Rasouli ${ }^{1,2}$

1. Social Determinants of Health Research Center, Research Institute for Health Development, Kurdistan University of Medical Sciences, Sanandaj, Iran

2. Clinical Research Development Center, Kowsar Hospital, Kurdistan University of Medical Sciences, Sanandaj, Iran

3. Center for Communicable Disease Control, Ministry of Health and Medical Education, Tehran, Iran

Correspondence Author: Bakhtiar Piroozi, Facuty of Medicine, Kurdistan University of Medical Sciences, Pasdaran Ave, Sanandaj, Iran Tel: +98 9108161432; (E-mail: bpiroozi@gmail.com) 


\begin{abstract}
Background: Physical inactivity (PI) is a public health concern around the world that has been growing with the advent of technology. It has often been studied in adults. This study aims to determine the prevalence of physical inactivity in Iranian children aged 10-12 years and the impact of socioeconomic inequality on it.
\end{abstract}

Methods: In this descriptive study, the study population consisted of 10-12 years old children from an Iranian population from Kurdistan, Fars and Markazi provinces in 2018. The sample size was 1590 individuals. The sampling method was cluster sampling. Data was collected using demographic questionnaire, modifiable activity questionnaire (MAQ) and socioeconomic questionnaire. Linear and logistic regression were used to estimate the final model and the Oaxaca analysis method was applied. All analyses were performed in Stata/SE 14.0.

Results: Of the 1590 participants, $52.82 \%$ were male. The results showed that $25.79 \%$ of the subjects had physical inactivity and $7.30 \%$ had moderate physical activity during the week. The average physical activity during one week was more in boys than in girls $(\mathrm{P}$-value<0.05). Children of mothers with secondary and high school education were more likely to have physical inactivity than mothers with a high school diploma or higher (AOR: 1.35, 95\% CI: $1.02-1.77$ ). The concentration index was -.11, indicating a greater concentration of physical inactivity in children with low socioeconomic levels.

Results: One-fourth of the study population had physical inactivity in this age group and required serious interventions. Socioeconomic levels, parental literacy, and children's sex were factors affecting the level of physical activity. Raising parental awareness, designing specific programs for girls, such as increasing access to sports facilities, are strategies to reduce physical inactivity in children. 
Keywords: Socioeconomic status, Inequalities, Physical inactivity, Concentration index, Iran

\section{Introduction}

Physical inactivity (PI) is a public health concern that is considered a potential risk factor for adverse health outcomes worldwide. The World Health Organization (WHO) reports the prevalence of PI to be more than $80 \%$ in adults and $23 \%$ in adolescents. This rate varies considerably across countries. Reports indicate that the prevalence of PI is higher in the eastern Mediterranean region, the US, Europe and the Western Pacific region than in other parts of the world (1). Urban and industrial life, advances in technology, economic development and globalization have led to rapid changes in lifestyle and PI in individuals, leading to an increase in the prevalence of related chronic diseases such as coronary heart disease, colon cancer, hypertension, stroke, breast cancer, type 2 diabetes and osteoporosis (2).

Studies show that many behavioral risk factors for chronic diseases, including PI, are rooted in childhood and that the patterns of behavior developed during this period remain relatively stable in adolescence and adulthood $(4,3)$. Therefore, it seems that children are an important target group for interventional and preventive activities. Any intervention in this group can be very effective in controlling the chronic diseases epidemic in adulthood (5).

Today's children are less active than their peers decades ago. Watching television and computer games are known to be the main causes of PI in children. There are also other factors in Iran, such as living in small houses and apartments, the phenomenon of single children or the low number of children in each family, lack of appropriate sports facilities in the community and schools, high school pressure, preference of education than other aspects of life, disregard for sports at school and home and lack of security in the community that affect inactivity and lower activity of children and adolescents $(6,5)$. 
One of the most important health indicators that influence one's attitude, behavior, and exposure to risk factors is socioeconomic status (SES). There is evidence to suggest that the lower the SES of a person, the worse their health status. There is, in fact, a social gradient in health that moves from the top to the bottom of the socioeconomic spectrum and creates gaps in health outcomes such as infection rates, mortality and disability across different social classes worldwide (7). In the 2014 study by Finger et al., the physical activity and SES of parents of German children aged 11-17 years were examined. The results showed that higher education of parents was positively correlated with the level of physical activity of children (8). Rey-López's study (2012) had similar results; its results showed an inverse relationship between socioeconomic factors such as parental education and child PI (9). A systematic review was also conducted to investigate socioeconomic factors affecting physical activity by Ragna Stalsberg et al. The results indicated a positive relationship between socio-economic factors and physical activity, especially the impact on leisure time (10).

To our knowledge, there is little information available on the status of physical activity in children in Iran. So far, very few studies have been conducted to investigate the effect of socioeconomic inequalities on PI in Iranian children. Therefore, the aim of the present study was to determine the prevalence of PI in Iranian children aged 10-12 years and the effect of socioeconomic factors and economic inequalities on it.

\section{Methods}

\section{Study setting and participants}

The population of this cross-sectional study was 10-12 years old children in fourth, fifth and sixth grades of elementary school. The final sample size was 1578 individuals. The multi-stage sampling method involving systematic classification, clustering and random methods were used. 
For the first step, the provinces of Iran were divided into three geographical clusters of 9, 9 and 13 provinces. One province was randomly selected from each cluster including Kurdistan province in the west, Markazi in the center and Fars in the east. In the next step, one city was randomly selected in the selected provinces including Marivan in Kurdistan, Saveh in Markazi and Garash in Fars. In order to reduce the error, the sample size of each cluster was selected proportional to the size of that cluster. Six elementary schools (three for girls and three for boys) were randomly selected from the primary schools in each city and 526 samples (263 female students and 263 male students) were randomly selected from each of the 10 to 12 -year-old students (Grade 5 and 6). Prior to the study, written consent was obtained from all parents and trained public health experts were used to collect data. Questionnaires used in this study included demographic information questionnaire, modifiable activity questionnaire (MAQ) and socioeconomic status questionnaire which were completed by children and parents at home based on the protocol.

In the MAQ questionnaire, each physical activity was weighted according to its metabolic equivalent (MET). One MET was the amount of energy consumed by a resting person every minute. Then, the level of physical activity was calculated in terms of hours of activity per week (MET-h/WK) and the subjects were divided into three groups based on their overall physical activity: light activity (MET<3), moderate activity (MET=3-6) and heavy activity (MET>6). Based on the studies, the reliability of this questionnaire is 0.97 and its validity is $0.47(12,11)$. To determine socioeconomic status, questions were asked about parental assets.

\section{Statistical Analysis}

Curve concentration, index concentration and odds ratio methods were used to measure inequality. The method proposed by O'Donnell et al. (2006) was used to calculate 
socioeconomic status. Accordingly, a socioeconomic status questionnaire consisting of a number of assets was used. Principal component analysis (PCA) first identified the variables that had the most impact on the variance of all variables and then a new socioeconomic status (SES) variable was constructed based on these variables. The PCA gives each asset a specific coefficient or weight then extracts a linear combination of the variables with the most variance. It then eliminates this variance and finds the second linear combination that describes the largest proportion of the residual variance, and continues this procedure. In this study, we developed an asset index using the PCA method. Five quintiles were constructed using the mean of this variable which divided the statistical population into 5 groups: very poor, poor, middle, rich and very rich. Finally, the PI variable was compared between the two very poor and very rich groups. Logistic regression was used to evaluate the relationship between prevalence of outcome variables in different socioeconomic subgroups. The highest socioeconomic group was considered as the baseline. The model began with the baseline model and then sequentially added the other determinants and explained the effect of each of the determinants contributing to inequality.

Concentration index (CI) and concentration curve (CC) methods were used to measure inequality. The CI quantitatively shows the degree of inequality at the income distribution level of a health variable. To calculate the relative CI, individuals are first sorted by socioeconomic status, and then the cumulative percentage of the population is plotted against a cumulative percentage of the health variable (enjoyment rate) to create the CC. CI values vary from +1 to -1 . It is one of the most common indices in calculating income inequality and socioeconomic status. Negative values indicate that the PI variable is concentrated among people with poor socioeconomic status and the $\mathrm{CC}$ is above the equality line. But positive values indicate that the 
PI variable is concentrated among the rich, and when the distribution of health among all individuals is the same, the CI will be zero. The Kakwani method was used to calculate the CI based on the following formula:

Formula 1.

$$
C=\frac{2}{\mu} \operatorname{Cov}\left(y_{i}, R_{i}\right)
$$

In this formula, $\mathrm{C}$ is the concentration index, $\mathrm{Cov}$ is covariance, yi is health variable, $\mathrm{Ri}$ is economic rank, and $\mu$ is mean health variable. Due to the limitations of this index for binary data, CI was normalized based on the method used by Wagstaff according to the following formula in which $\mathrm{C}$ is the standard concentration index and $\mu$ is mean health variable.

Formula 2. Wc $=\mathrm{C} /(1-\mu)$

After measuring the inequalities, the decomposition concentration index was used to determine the contribution of each of the determinants to inequality. According to the Wagstaff method, we considered a linear regression model in relation with PI variable (y) and a set of determinants $\left(x_{k}\right)$ :

$y_{i}=\alpha+\sum_{k} \beta_{k} x_{k i}+\varepsilon_{i} \quad$ Formula 3.

$\mathrm{i}$ is the average $\mathrm{i}^{\text {th }}$ person, $\beta_{k}$ is the regression coefficient, $\varepsilon_{i}$ is error coefficient or interpersonal changes. Based on the relationship between $y_{i}$ and $x_{k}$ in formula 3, we write $\mathrm{C}$ for PI as follows:

$$
C=\sum\left(\frac{\beta_{k} \bar{x}_{k}}{\mu}\right) C_{k}+\frac{G C_{\varepsilon}}{\mu} \quad \text { Formula } 4
$$

In this formula, inequality generally consists of two explained and unexplained parts. In the explained part, $\mu$ is the mean health variable, $\beta_{k}$ is the regression coefficients for PI on the available determinants, $\bar{x}_{k}$ is the mean of the determinants or $x_{k}, C_{k}$ is the concentration index 
$\mathrm{C}$ for the $x_{k}$ determinant and in the unexplained part, $\frac{G C_{\varepsilon}}{\mu}$ is the residual error in the total $\mathrm{C}$ for $\varepsilon_{i}$.

In this study, PI was considered as the response variable and Chi-square test was used to estimate the prevalence of response variable at each level of demographic variables. Multivariate logistic regression was used to estimate the final model based on variables with $p<0.1$ in chi-square test and calculation of univariate OR and AOR. All analyses were performed in Stata/SE 14.0.

\section{Results}

Of the 1590 participants, $410(25.79 \%)$ were physically inactive, $116(7.30 \%)$ had moderate physical activity and 1064 (66.92\%) had heavy physical activity during the week.

Among the participants, $613(38 \%)$ went running, 199 (12\%) did martial arts, $116(7 \%)$ gymnastics and endurance sports, $633(40 \%)$ football or handball, 100 (6\%) basketball, 485 (30\%) volleyball, 696 (44\%) cycling, 133 (8\%) ping pong or badminton, (2\%) bodybuilding or weightlifting, $347(22 \%)$ swimming, 78 (5\%) wrestling, 8 (0.05\%) skiing, 212 (13\%) skating, $226(14 \%)$ hiking or outings, $124(7 \%)$ dance or rhythmic movements, and 90 people (5\%) did other physical activities.

The mean (standard deviation) of total physical activity in terms of (MET-h/WK) was 40.72 (76.2). The mean (SD) of physical activity among girls was 30.87 (58.44) and among boys was 49.61 (88.28) MET hours per week. The mean (SD) of physical activity among fourth-graders was 40.1 (77.9), fifth graders 38.7 (64.7) and sixth graders 43.5 (88.3). Table 1 shows physical activity in terms of sex, educational grade, BMI, maternal education, age of father, age of mother, highest education level of both parents, and household size. Mean physical activity during one week was significantly different between boys and girls ( $\mathrm{P}$-value $<0.05)$. 
In Table 2, univariate OR is presented for those variables in Table 1 that had a significant relationship at $\mathrm{P}$-value $=0.2$ with physical activity level, using univariate and multivariate logistic regression. The OR for the variables of sex and maternal education had a significant relationship with physical activity level (P-value<0.05). In multivariate logistic regression, sex and maternal education at secondary and high school level had a significant relationship with physical activity level (P-value $<0.05)$.

Also, the concentration index for PI was negative $(\mathrm{CI}=-0.11)$, indicating inadequate activity in children with low socioeconomic status. The CC of PI is above the equality line, indicating a greater concentration of PI in the poorer group of the community (Fig 1).

Table 3 shows the results of CI decomposition for PI in children. The first column shows the partial effect regression coefficients for each determinant on the outcome variable (PI) in the presence of other variables. The second column indicates the elasticity of PI and the third column shows the CI for each determinant. The positive or negative sign indicates the concentration of the relevant determinant in the rich or poor group. The last two columns show the absolute contribution value and the CI of each detriment relative to the overall CI. The positive (negative) absolute value of a determinant's contribution indicates its contribution to the pro-poor (pro-rich) inequality. The reference group of 10-year-old male students with parents under 35 years of age and high school diploma or academic education was considered the highest economic class and household size of less than 4 children. Unfavorable economic situation ($537.41 \%)$, father's education (385.87\%) and household size (123.11\%) respectively had the greatest role in creating inequalities in PI. The contribution of mother's education in creating inequality was -181.17 . The contribution of parents' age in creating inequality at age 35 to 44 was 45.39 and 8.96, respectively, and the contribution of age was -52.85 (Table 3). 


\section{Discussion}

Over $25 \%$ of children had PI. The situation was even worse for girls. Children with more educated mothers had better physical activity. PI was less favorable in people with lower socioeconomic status.

PI is one of the most important risk factors for mortality worldwide. It is one of the pests of industrial life that is increasing every year due to advances in science and technology (13).

The results showed that about one quarter of children in the study had PI, indicating an undesirable condition. The results of other studies conducted in Iran and other parts of the world also show a high prevalence of PI in children all over the world (14-16). The results of the study by Kelishadi et al. on 6-18 years children showed that $24 \%$ of them were physically inactive and the average physical activity in boys was higher than in girls, which is consistent with the present study (16). A systematic review to assess PI in Arab countries also found that the prevalence of PI was higher in girls than in boys (17). Differences in physical activity can be influenced by gender-defined roles or societal norms and values, which may result in women having less access to resources for exercise and have fewer choices for leisure time $(19,18)$. Also, differences in psychological and behavioral aspects can be effective in making these differences (20).

In the present study, maternal literacy level was identified as an effective factor on PI of children. The findings showed that children with mothers with low levels of literacy had a higher chance of PI than others. Mothers with more education appear to be more aware of the importance of regular physical activity in children $(22,21)$. However, the results of some studies are different and show that the level of physical activity in children decreases as mothers' education and employment levels increase (23). These differences may be justified by different 
cultural and family backgrounds. Providing proper awareness and education about the necessity of physical activity in children is one of the critical issues that should be considered for families. One message of this study to improve physical activity is to increase mothers' education level. Another determinant of health and well-being in all periods of life is socio-economic status, which is considered an important factor in creating health inequalities in populations (24). In this study, the factors affecting inequality were investigated using the decomposition model. The findings showed that low economic status, level of education of the father and the family size were the most important factors in creating inequality. CI analysis showed that people with low economic status had less physical activity than other children. These children may not have access to sports facilities and equipment due to their cost constraints or may be reluctant to do so due to lack of time and lack of access to safe and appropriate sports facilities (25). A study by Prins et al. on Dutch adolescents showed that adolescents' lack of involvement in sport activities was highly correlated with lack of access to sports venues, parks and green spaces, and the socioeconomic status of households in a neighborhood (26). On the other hand, some scholars believe that people in the upper classes are most aware of the goals of a regular physical activity promotion program $(28,27)$. However, some studies have had different results and have shown that children in the upper classes may have less physical activity for reasons such as having a more prosperous life, more consumption of fast foods, more use of vehicles, computer games, mobile phones, etc. $(30,29)$.

In the present study, fathers' education level also played an important role in creating inequalities: children of fathers with low educational level were more inactive. This difference may be due to the low level of literacy, awareness and attitude of the head of household towards the benefits of physical activity of children and not providing suitable conditions for physical 
activity in children according to their life preferences. It seems that when education level of parents increases, they can better encourage their children to do physical activity. Similar results were obtained in the Eduardo Gonzalo-Almorox study, which examined the effect of socioeconomic inequalities on leisure-time inactivity of Spanish children. The results of this study showed that the education level of household head and family income were the main socioeconomic inequalities affecting child inactivity (31). Also, in the present study, household size or the number of individuals in the family was one of the factors affecting the inactivity of children. Children with more than 4 family members had more PI than families with less than 4 members. In a study carried out in Kurdistan in 2014, the factors affecting inequality in PI of children aged 10-12 were investigated using Oxaca model. The results of the study showed that the PI of individuals in the lower socioeconomic classes was higher than the others. In the initial model, increased maternal literacy, improved socioeconomic status, and improved living place were all effective in increasing physical activity. In the final Oxaca model, it was also found that family life in slums and lower literacy of mothers accounted for most of the differences in children's physical activity (32). These differences could be due to cultural differences or other contextual factors. But it seems that, given the contradictions in the results of various studies, education on the importance of public health and adopting healthy behaviors during childhood and adulthood due to the dangers of inadequate physical activity are essential to have a healthy community away from economic and social differences. School-based interventions, with the involvement of students' families, and multidisciplinary interventions can ultimately lead to increased physical activity in children and adolescents $(34,33)$.

In the present study, cycling and sports such as soccer or handball were more common than other physical activities, and skiing accounted for the lowest percentage of physical activity in 
children. This difference, on the one hand, may be due to the availability of sports facilities for both sexes, the costs and seasonality of a sport, and, on the other hand, due to parents' views on its impact on children's future. These results indicate that boys and girls have different expectations and interests in choosing their physical activities, which is very important for policy-making and planning.

One of the limitations of this study may be recall bias due to questions about subjects' activities in a past period, which were attempted to be reduced as much as possible by training and protocol preparation. However, the present study with a large sample size provides valuable evidence on family and behavioral risk factors affecting PI in Iranian children, which can justify further related studies, effective interventions, and planning and policy making.

\section{Conclusion}

One quarter of the study population had PI, which is alarming for this age group. The situation of the girls was worse. PI was more concentrated in children with poorer socioeconomic status. Socioeconomic levels, parental literacy, and children's sex were factors affecting the level of physical activity. Improving the socioeconomic status of households, increasing the education of parents and especially mothers, and paying more attention to groups with lower socioeconomic status are recommended to improve physical activity in children. Large-scale national policies are recommended to promote physical activity in children.

\section{Abbreviations}

PI: Physical inactivity; MAQ: Modifiable activity questionnaire; SES: socioeconomic status; PCA: Principal component analysis; SD: standard deviation

\section{Acknowledgments}

The authors would like to thank all participants for help us to perform this study. 


\section{Funding}

This study was funded by Vice Chancellor for Research and Technology of Kurdistan University of Medical Sciences, Sanandaj, Iran. The funding body played no role in the design of the study, collection, analysis, or interpretation of data or in writing the manuscript.

\section{Availability of data and materials}

The datasets used and/or analyzed during the current study can be made available by the corresponding author on reasonable request.

\section{Authors' contributions}

GM and BP conceived and designed the study. BZ, FM, BP and GM analyzed and interpreted the data, and drafted the manuscript. GM, BP, MM, BZ and MAR were involved in the composition of the study tool, supervision of the research process and critical revision and review of the manuscript. All the authors read and approved the final manuscript.

\section{Ethics approval and consent to participate}

Parents in all participating families provided written, informed consent and children provided verbal assent at each measurement occasion. The approval for this study was obtained from the Ethics Committee of Kurdistan University of Medical Science (IR.MUK.REC.1396.164).

\section{Consent for publication}

Not applicable.

\section{Competing Interests}

The authors have no conflicts of interest to declare. 


\section{References}

$.1 \quad$ World Health Organization. Global Strategy on Diet, Physical Activity and Health 2015 [Available from: http://www.who.int/dietphysicalactivity/pa/en/.

.2 Humphreys BR, McLeod L, Ruseski JE. Physical activity and health outcomes: evidence from Canada. Health economics. 2014;23(1):33-54.

.3 Telama R, Yang X, Viikari J, Välimäki I, Wanne O, Raitakari O. Physical activity from childhood to adulthood: a 21-year tracking study. American journal of preventive medicine. 2005;28(3):267-73.

.4 Goran M, Hunter G, Nagy T, Johnson R. Physical activity related energy expenditure and fat mass in young children. International journal of obesity. 1997;21(3):171.

.5 Gao Z, Chen S, Sun H, Wen X, Xiang P. Physical activity in children's health and cognition. BioMed research international. 2018;2018.

.6 Zhang T, Wang P, Liu H, Wang L, Li W, Leng J, et al. Physical activity, TV watching time, sleeping, and risk of obesity and hyperglycemia in the offspring of mothers with gestational diabetes mellitus. Scientific reports. 2.1-9:(1)7;017

.7 Pulsford RM, Griew P, Page AS, Cooper AR, Hillsdon MM. Socioeconomic position and childhood sedentary time: evidence from the PEACH project. International Journal of Behavioral Nutrition and Physical Activity. 2013;10(1):105.

.8 Finger JD ,Mensink GB, Banzer W, Lampert T, Tylleskär T. Physical activity, aerobic fitness and parental socio-economic position among adolescents: the German Health Interview and Examination Survey for Children and Adolescents 2003-2006 (KiGGS). International Journal of Behavioral Nutrition and Physical Activity. 2014;11(1):43. 
.9 Rey-López JP, Tomas C, Vicente-Rodriguez G, Gracia-Marco L, Jiménez-Pavón D, Pérez-Llamas F, et al. Sedentary behaviours and socio-economic status in Spanish adolescents: the AVENA study. European Journal of Public Health. 2010;21(2):151-7.

.10 Stalsberg R, Pedersen AV. Are differences in physical activity across socioeconomic groups associated with choice of physical activity variables to report? International journal of environmental research and public health. 2018;15(5):922.

.11 Mocanu V. Prevalence of overweight and obesity in urban elementary school children in northeastern Romania: its relationship with socioeconomic status and associated dietary and lifestyle factors. BioMed research international. 2013;2013.

.12 Shailee F, GM S, KR S. Association between Dental caries and body mass index among 12 and 15 years school children in Shimla, Himachal Pradesh. Journal of Advanced Oral Research. 2013;4(1):6-10.

.13 Anderson E, Durstine JL. Physical activity, exercise, and chronic diseases: A brief review. Sports Medicine and Health Science. 2019;1(1):3-10.

.14 Peltzer K, Pengpid S. Leisure time physical inactivity and sedentary behaviour and lifestyle correlates among students aged 13-15in the association of Southeast Asian nations (ASEAN) member states, 2007-2013. International journal of environmental research and public health. 2016;13(2):217.

.15 Bloemers F, Collard D, Paw MCA, Van Mechelen W, Twisk J, Verhagen E. Physical inactivity is a risk factor for physical activity-related injuries in children. British journal of sports medicine. 2012;46(9):669-74.

.16 Kelishadi R, Qorbani M, Djalalinia S, Sheidaei A, Rezaei F, Arefirad T, et al. Physical inactivity and associated factors in Iranian children and adolescents: the Weight Disorders 
Survey of the CASPIAN-IV study. Journal of cardiovascular and thoracic research. 2017;9(1):41.

.17 Sharara E, Akik C, Ghattas H, Obermeyer CM. Physical inactivity, gender and culture in Arab countries: a systematic assessment of the literature. BMC public health. 2018;18(1):639.

.18 Forthofer M, Dowda M, O’Neill JR, Addy CL, McDonald S, Reid L, et al. Effect of child gender and psychosocial factors on physical activity from fifth to sixth grade. Journal of physical activity and health. 2017;14(12):953-8.

.19 Hatamzadeh Y, Habibian M, Khodaii A. Walking behavior across genders in school trips, a case study of Rasht, Iran. Journal of Transport \& Health. 2017;5:42-54.

.20 Blatchford P, Baines E, Pellegrini A. The social context of school playground games: Sex and ethnic differences, and changes over time after entry to junior school. British Journal of Developmental Psychology. 2003;21(4):481-505.

.21 Hesketh K, Crawford D, Salmon J. Children's television viewing and objectively measured physical activity: associations with family circumstance. International Journal of Behavioral Nutrition and Physical Activity. 2006;3(1):36.

.22 Kestilä L, Mäki-Opas T, Kunst AE, Borodulin K, Rahkonen O, Prättälä R. Childhood adversities and socioeconomic position as predictors of leisure-time physical inactivity in early adulthood. Journal of physical activity and health. 2015;12(2):193-9.

.23 Sherar LB, Griffin TP, Ekelund U, Cooper AR, Esliger DW, van Sluijs EM, et al. Association between maternal education and objectively measured physical activity and sedentary time in adolescents. J Epidemiol Community Health. 2016;70(6):541-8. 
.24 O’Donoghue G, Kennedy A, Puggina A, Aleksovska K, Buck C, Burns C, et al. Socioeconomic determinants of physical activity across the life course: A" DEterminants of DIet and Physical ACtivity”(DEDIPAC) umbrella literature review. PLoS One. 2018;13(1).

.25 Henriksen PW, Rayce SB, Melkevik O, Due P, Holstein BE. Social background, bullying, and physical inactivity: National study of 11- to 15-year-olds. Scandinavian Journal of Medicine \& Science in Sports. 2016;26(10):1249-55.

.26 Prins RG, Mohnen SM, van Lenthe FJ, Brug J, Oenema A. Are neighbourhood social capital and availability of sports facilities related to sports participation among Dutch adolescents? International Journal of Behavioral Nutrition and Physical Activity. 2012;9(1):90.

.27 Bauman AE, Reis RS, Sallis JF, Wells JC, Loos RJ, Martin BW, et al. Correlates of physical activity: why are some people physically active and others not? The lancet. 2012;380(9838):258-71.

.28 Kohl 3rd HW, Craig CL, Lambert EV, Inoue S, Alkandari JR, Leetongin G, et al. The pandemic of physical inactivity: global action for public health. The lancet. 2012;380(9838):294305.

.29 Cottrell L, Zatezalo J, Bonasso A, Lattin J, Shawley S, Murphy E, et al. The relationship between children's physical activity and family income in rural settings: A cross-sectional study. Preventive medicine reports. 201.2:99-104;5

.30 Kelishadi R, Qorbani M, Motlagh ME, Ardalan G, Heshmat R, Hovsepian S. Socioeconomic disparities in dietary and physical activity habits of Iranian children and adolescents: The CASPIAN-IV study. 2016. 
.31 Gonzalo-Almorox E, Urbanos-Garrido RM. Decomposing socio-economic inequalities in leisure-time physical inactivity: the case of Spanish children. International journal for equity in health. 2016;15(1):106.

.32 Mostafavi F, Moradi G, Azadi N, Esmaeilnasab N, Roshani D. Using oaxaca decomposition to study socioeconomic inequity of physical activity among children aged 10-12 years: A study in West of Iran. International journal of preventive medicine. 2019;10.

.33 Nicksic NE, Salahuddin M, Butte NF, Hoelscher DM. Associations between parentperceived neighborhood safety and encouragement and child outdoor physical activity among low-income children. Journal of Physical Activity and Health. 2018;15(5):317-24.

.34 Brusseau TA, Hannon J, Burns R. The effect of a comprehensive school physical activity program on physical activity and health-related fitness in children from low-income families. Journal of Physical Activity and Health. 2016;13(8):888-94. 
Fig. 1. The concentration curve for physical Inactivity in children in Iran

Table 1. Physical inactivity in terms of demographic variables

Table 2. Univariate and multivariate logistic regression of physical inactivity

Table 3. Decomposition of concentration results for physical inactivity 
Figures

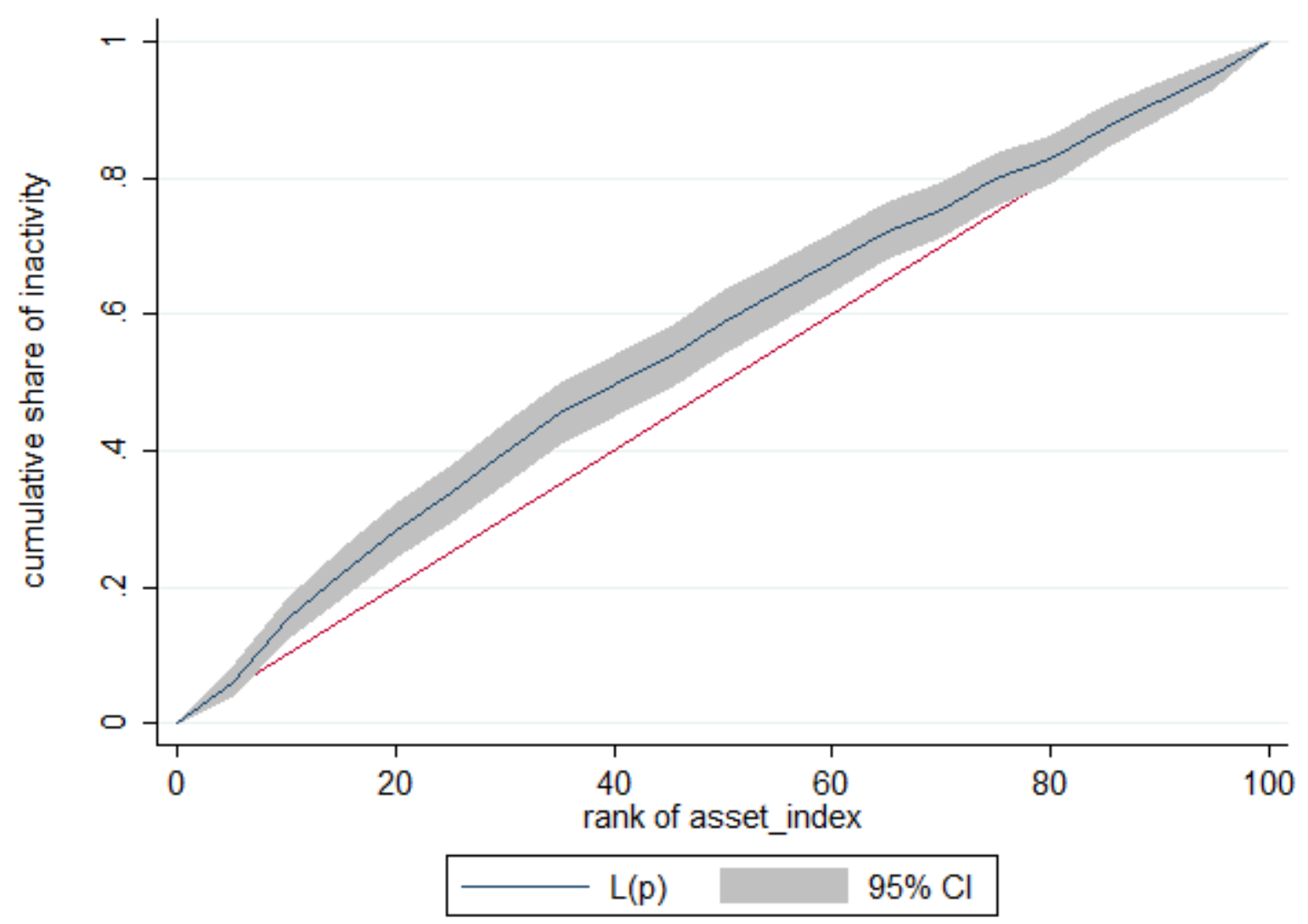

Figure 1

The concentration curve for physical Inactivity in children in Iran 